\section{COLICINS}

\section{Killer Proteins in Action}

from our Cell Biology Correspondent

LIKE an outsider coming from behind the field and forcing the favourite to a photo finish and a dead heat, Senior and Holland report, in the latest issue of the Proceedings of the US National Academy of Sciences (68, 959; 1971), that colicin E3 acts by activating a specific ribonuclease in sensitive Escherichia coli which cleaves a fragment from the $3^{\prime}$ terminal end of the 16S RNA of the 30 S ribosomal subunit and so inactivates the ribosome. In the same issue of the Proceedings, indeed in the very next paper (ibid., 964), Bowman, Dahlberg, Ikemura, Konisky and Nomura report identical conclusions.

When not otherwise occupied in reconstructing the large and small ribosomal subunits of $E$. coli from their constituent nucleic acids and proteins, Nomura has spent much time deciphering the biology of colicins, in particular colicin E3. It was he and his coworkers who showed that these protein molecules, which are specified by extrachromosomal genetic elements in certain strains of $E$. coli, can adsorb to the surfaces of susceptible $E$. coli and, without penetrating the surface, rapidly inhibit protein synthesis without at the same time affecting nucleic acid metabolism. Moreover, it was Nomura who proved that ribosomes isolated from susceptible $E$. coli, after exposure to colicins, are less able to support polypeptide synthesis in vitro than ribosomes from untreated cells and that this defect resides in the small $30 \mathrm{~S}$ ribosomal subunit.

The mystery of these observations, which the two groups have now solved, was simply the question of how a protein which only binds to the outside surface of a bacterium can cause the specific inactivation of the $30 \mathrm{~S}$ ribosomes of that cell. The only plausible hypothesis was that by binding to the bacterium's surface the colicin causes some conformational change in the cell membrane. The inactivation of $30 \mathrm{~S}$ ribosomes might then ensue if the conformational change activates either a ribonuclease or a protease which could modify and inactivate the ribosomal RNA or some crucial ribosomal protein.

Pursuing this line of argument, Bowman et al. isolated the proteins and RNA from 30S ribosomes of bacteria exposed to colicin E3 and from untreated bacteria. These were then used in a series of reciprocal reconstructions of the $30 \mathrm{~S}$ ribosome and the reconstructed particles were tested for their ability to support protein synthesis. Such a mammoth task, which only
Nomura's group is equipped to do, proved that the defect in the $30 \mathrm{~S}$ ribosomes of cells exposed to colicin E3 resides in the $16 \mathrm{~S}$ ribosomal RNA and not in the complement of ribosomal proteins. Bowman et al. therefore made fingerprints of the $16 \mathrm{~S}$ ribosomal RNA from cells treated with colicin E3 and compared them with fingerprints of 16S RNA from untreated cells. There were several oligonucleotides missing from the former RNA, including the oligonucleotide which contains the normal $3^{\prime}$ terminal base of $16 \mathrm{~S}$ ribosomal RNA.

Not satisfied at leaving things at that stage, Nomura's group successfully isolated a fragment of about fifty bases from cells exposed to colicin E3. On fingerprinting, this fragment was found to yield the $3^{\prime}$ terminal and other missing oligonucleotides. It is the piece of RNA cleaved from the $16 \mathrm{~S}$ ribosomal RNA by the specific ribonuclease which must be activated when colicin E3 binds to the cell surface.

Although not able to do the elegant ribosome reconstruction experiments of Nomura and his colleagues, Senior and Holland analysed by gel electrophoresis the 16S RNA and proteins of the 30S ribosomes from cells exposed and not exposed to colicin E3. The only differences they could detect were with the 16S RNA. Further analysis of this RNA from cells exposed to colicin E3 showed, in fact, that it sediments at 15S, not $16 \mathrm{~S}$, and fingerprints show that this 15S RNA lacks the $3^{\prime}$ terminal sequence of intact 16S RNA.

All that now remains to complete this quite remarkable story of how colicin E3 works is the identification of the location and nature of the ribonuclease, which is activated when this colicin binds to cells and which is so specific for some sequence in $16 \mathrm{~S}$ ribosomal RNA.

\title{
Magnetic Fields in Radio Sources
}

THE intense signals from most galactic and extragalactic radio sources are thought to be produced by the synchrotron process in which relativistic electrons gyrate in the source magnetic fields. Although the magnetic field structure has been derived from many sources from measurements of linear polarization, it has proved difficult to obtain reliable estimates of the magnitude of these fields. Arguments based on an assumed equipartition between the energy of relativistic particles and magnetic fields suggest an order of magnitude for the fields of $10^{-4}$ gauss. A more direct assessment of the field strength can be obtained from investigations of the synchrotron self-absorption in a source, but this requires measurements of the radio flux over a wide frequency range as well as interferometer observations, usually on intercontinental baselines.

A simple and potentially powerful technique for measuring source magnetic fields is now emerging based on a determination of the percentage circular polarization. Several years ago, theoretical calculations by Sciama and Rees (Nature, 216, 147 ; 1967) and by Legg and Westfold (Astrophys. J., 154, $499 ; 1968)$ showed that the synchrotron process would give weak but significant circular polarization which present-day experimental techniques can detect. Observers now agree that the circular polarization of about one per cent which is emitted by the planet Jupiter indicates the presence of a field of 0.4 gauss in its van Allen belts. In extra- galactic sources of high emissivity, Conway, Gilbert, Raimond and Weiler (Mon. Not. Roy. Astron. Soc., 152, 1p ; 1971) believe there is significant circular polarization of a few tenths of a per cent which indicates fields of up to $10^{-3}$ gauss.

In next Monday's Nature Physical Science, E. R. Seaquist of the David Dunlap Observatory, Ontario, Canada, reports a detailed study of the $\mathrm{z}=1.187$ quasi-stellar object PKS 1127-14 made with the 46-metre telescope of the Algonquin Radio Observatory. $\mathrm{He}$ finds significant circular polarization which varies on a time scale of 1.5 years and has a maximum value of one per cent. Synchrotron emission theory indicates that the magnetic field in PKS $1127-14$ would be about $6 \times 10^{-2}$ gauss. The importance of this result is that it can be checked against the many other observations of this source over a wide range of wavelengths which can be used to build up a good picture of its physical properties. Measurements of the variability of PKS $1127-14$ show that its small diameter $(\leqslant 0.003$ seconds of arc) component also has a time scale of 1.5 years. This same component has a maximum in its spectrum at 7,000 $\mathrm{MHz}$ which suggests a magnetic field strength comparable with that indicated by the circular polarization measurements. The results look very promising and should stimulate more extensive observations and calculations of the circular polarization from more realistic magnetic configurations of radio sources. 